\title{
Holographic storage dynamics in lithium niobate: theory and experiment
}

\author{
Amnon Yariv and Sergei S. Orlov \\ T. J. Watson Laboratory, California Institute of Technology, Pasadena, California 91125 \\ George A. Rakuljic \\ Arroyo Optics, Inc., Santa Monica, California 90404
}

Received January 2, 1996; revised manuscript received May 31, 1996

\begin{abstract}
We present a theoretical model that describes holographic ionic fixing and storage dynamics in photorefractive crystals. Holographic gratings that are based on charge redistribution inevitably decay because of ionic and electronic conduction. Relevant decay rates and transient hologram field expressions are derived. Ionic gratings are partially screened by trapped electrons on readout. The lifetimes of fixed ionic holograms are limited by the finite ionic conductivity at low (i.e., room) temperatures. Only under certain and restricted conditions can these decay times be acceptably long. A significant increase in fixed ionic hologram lifetime is realized in lithium niobate with a low hydrogen-impurity content. The residual ionic conductivity (decay-time constant) in these samples exhibits $\sim 1.4-\mathrm{eV}$ activation energy and is not due to protonic conduction. Fixed hologram lifetimes of $\sim 2$ years at room temperature in dehydrated lithium niobate crystals are projected. (C) 1996 Optical Society of America.
\end{abstract}

\section{INTRODUCTION}

Holographic data storage in photorefractive crystals is a topic of intense current interest. ${ }^{1-3}$ It is driven by the prospects of the large storage capacity, $\sim V / \lambda^{3}$ bits in a volume $V$. Two of the important concerns in this field involve the lifetimes of fixed and developed holograms and their strength. Volume phase holograms in photorefractive materials are produced by the redistribution of photoexcited carriers (e.g., electrons) in the presence of light. To avoid fast erasure of the holograms on readout several techniques have been developed. ${ }^{4-7}$ In general, efficient ionic fixing is based on the great disparity between the dark electronic conductivity at elevated temperatures and the ionic conductivity. At elevated temperatures the ionic conductivity is dominant, and ions readily compensate for the holographic electric field pattern created by photoexcited electrons by mimicking their spatial distribution. At low temperatures the ionic conductivity is also low, permitting the quasi-permanent storage of the ionic replica of the initial electronic hologram. The residual ionic conductivity at low temperature thus determines the lifetime of the fixed hologram.

A typical history of a hologram is sketched in Fig. 1. In phase I an electronic grating, previously recorded, is heated to cause ionic transport. This leads to a compensated grating, which is represented by the net space charge field amplitude $E_{1}^{(1)}$. In phase II the grating is left in the dark or is exposed to infrared light, which is not photoactive. This stage corresponds to a slow dark decay of both the electronic grating and the ionic compensating grating, which adiabatically follows the former. In many applications, however, the hologram is exposed to a reading light as in phase III. This light causes a partial re- distribution of the trapped electrons, culminating in a quasi-stable field $E_{1}^{(2)}$. This field will proceed to decay to zero under illumination because of ionic transport (phase IV).

The problem of grating dynamics has been considered extensively. ${ }^{8-14}$ What differentiates our study is the fact that, by taking advantage of the great disparity between the ionic and the electronic transport time constants that are involved in typical crystals, especially lithium niobate $\left(\mathrm{LiNbO}_{3}\right)$, we are able to obtain simple analytic expressions $^{9}$ for the time-dependent field in each of the phases defined above under realistic conditions.

\section{THEORETICAL MODEL}

\section{A. Two-Species Conduction Formulation}

In the model we consider both the conduction of the optically excited electrons and the ionic transport as well as the thermally activated dark electronic conductivity. Free conduction band electrons can be excited optically or thermally from a single-donor level, whereas ionic species are subject to drift owing to the internal space-charge fields and to diffusion owing to their own density gradients. We fully account for the photovoltaic currents, even when they are distributed inhomogeneously.

The dynamic variables are the mobile electron density $n_{e}$, the ion density $n_{i}$, and the trapped charge (ionized donor) density $N_{d}{ }^{+}$. The complete set of transport equations first formulated by Kukhtarev ${ }^{15}$ is

$$
\frac{\partial N_{d}^{+}}{\partial t}=\left(\sigma / h \nu I_{0}+\beta\right)\left(N_{d}-N_{d}^{+}\right)-\gamma_{e} N_{d}{ }^{+} n_{e},
$$




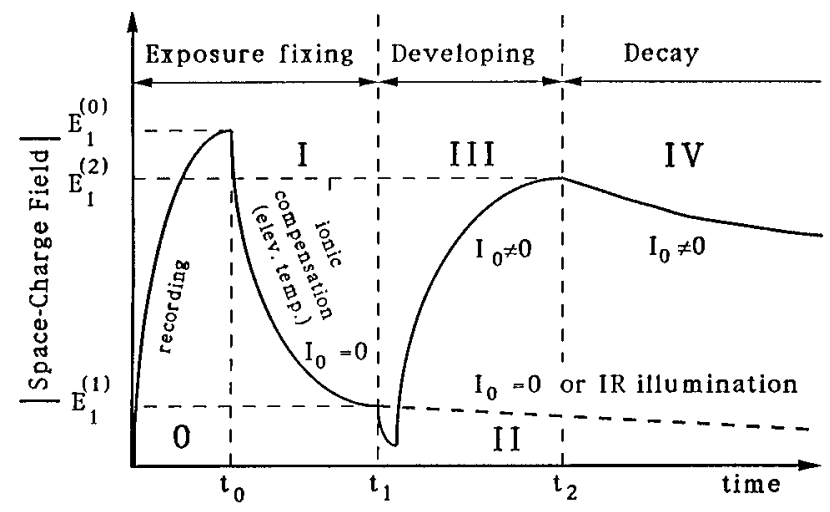

Fig. 1. Typical life history of a hologram in a photorefractive material. The Roman numerals are phase numbers.

$$
\begin{aligned}
\frac{\partial n_{e}}{\partial t}= & \frac{\partial N_{d}^{+}}{\partial t}+\frac{1}{e} \frac{\partial}{\partial x} \\
& \times\left[e \mu_{e} n_{e} E+e D_{e} \frac{\partial n_{e}}{\partial x}+\kappa \sigma I_{0}\left(N_{d}-N_{d}^{+}\right)\right], \\
\frac{\partial n_{i}}{\partial t}= & -\frac{1}{e} \frac{\partial}{\partial x}\left(e \mu_{i} n_{i} E-e D_{i} \frac{\partial n_{i}}{\partial x}\right), \\
\epsilon \epsilon_{0} \frac{\partial E}{\partial x}= & e\left(N_{d}^{+}-N_{a}+n_{i}-n_{i 0}-n_{e}\right) .
\end{aligned}
$$

In Eqs. (1), $I_{0}$ is the average optical intensity, $\sigma N_{a}{ }^{o}$ is the optical intensity absorption coefficient, $\beta$ is the thermal excitation rate, and $\gamma_{e}$ is the recombination constant for electrons. $\mu_{e}$ and $\mu_{i}$ are the mobilities of the electrons and ions, respectively, and we have assumed that $D / \mu$ $=k_{b} T / e$, where $k_{b}$ is the Boltzmann constant. $\kappa$ is the photovoltaic coefficient. The density of neutral donors is $N_{d}{ }^{o}$. Total donor density is $N_{d}=N_{d}{ }^{o}+N_{d}{ }^{+}$, and $N_{d o}{ }^{+}=N_{a}$ is the density of deep traps. The total current is equal to the sum of the ionic and electronic cur-
Using the above definitions, we can reduce transport equations (1) to the following linearized form:

$$
\begin{aligned}
\frac{\partial N_{d 1}{ }^{+}}{\partial t}= & -\left(\sigma / h \nu I_{0}+\beta\right) \frac{N_{d}}{N_{a}} N_{d 1}^{+}-\gamma_{e} N_{a} n_{e 1} \\
\frac{\partial n_{e 1}}{\partial t}= & -\left(\sigma / h \nu I_{0}+\beta\right) \frac{N_{d}}{N_{a}}+\frac{\mu_{e} n_{e 0} e}{\epsilon} \\
& \left.+i \frac{\kappa \sigma I_{0} K}{e}\right] N_{d 1}{ }^{+} \\
& -\left(\gamma_{e} N_{a}+\frac{\mu_{e} n_{e 0} e}{\epsilon}+D_{e} K^{2}+i \mu_{e} K E_{0}\right) n_{e 1} \\
& +\frac{\mu_{e} n_{e 0} e}{\epsilon} n_{i 1}, \\
\frac{\partial n_{i 1}}{\partial t}= & -\left(\frac{\mu_{i} n_{i 0} e}{\epsilon}+D_{i} K^{2}+i \mu_{i} K E_{0}\right) n_{i 1} \\
& +\frac{\mu_{i} n_{i 0} e}{\epsilon}\left(n_{e 1}-N_{d 1}^{+}\right) \\
E_{1}= & \frac{i e}{\epsilon K}\left(N_{d 1}^{+}+n_{i 1}-n_{e 1}\right)
\end{aligned}
$$

with Eq. (3d) following from the Gauss law.

A major feature of our analysis is the inequality $n_{e 1}$ $\ll N_{d 1}{ }^{+}, n_{\mathrm{i} 1}$, so that $n_{\mathrm{e} 1}$ can be neglected in Eqs. (3d) and (3c) but not otherwise. This inequality results from the fact that the electron trapping rate $\gamma_{e} N_{a} \sim 10^{7}-10^{9} \mathrm{~s}^{-1}$ exceeds by many orders of magnitude any other rate, which causes the electrons to reach an essentially instantaneous (i.e., within $t \leqslant 10^{-7} \mathrm{~s}$ ) local equilibrium with $N_{d 1}{ }^{+}$and $n_{i 1}$. We obtain the equilibrium value of $n_{e 1}$ by setting the left-hand side of Eq. (3b) equal to zero. This enabled us to eliminate $n_{e 1}$ from the mathematics (but not from the physics) and rewrite the governing equations of grating dynamics [(3a)-(3d)] in terms of two dynamic variables, i.e., $n_{i 1}$ and $N_{d 1}{ }^{+}$, only:

$\frac{\partial N_{d 1}{ }^{+}}{\partial t}=-N_{d 1}{ }^{+} \frac{\omega_{e} \gamma_{e} N_{a}+\left(\frac{\sigma}{h \nu} I_{0}+\beta\right) \frac{N_{d}}{N_{a}}\left(D_{e} K^{2}-i \mu_{e} K E_{0}\right)-i K \kappa \frac{\sigma}{h \nu} I_{0} \gamma_{e} N_{a}}{\gamma_{e} N_{a}+D_{e} K^{2}-i \mu_{e} K E_{0}}-n_{i 1} \frac{\omega_{e} \gamma_{e} N_{a}}{\gamma_{e} N_{a}+D_{e} K^{2}-i \mu_{e} K E_{0}}$,

rents and that which is due to the photovoltaic effect.

Equations (1) are linearized by approximating the dynamic variables in terms of (time and space) averages and small-signal time-varying amplitudes; i.e.,

$$
\begin{aligned}
n_{e} & =n_{e 0}+\left[n_{e 1} \exp (-i K x)+\text { c.c. }\right], \\
N_{d}{ }^{+} & =N_{a}+\left[N_{d 1}{ }^{+} \exp (-i K x)+\text { c.c. }\right], \\
n_{i} & =n_{i 0}+\left[n_{i 1} \exp (-i K x)+\text { c.c. }\right],
\end{aligned}
$$

where $n_{e 1} \ll n_{e 0}$, etc., $K$ is the spatial frequency of the grating, and $x$ is the spatial coordinate. A dependent variable is the space-charge field

$$
E=E_{0}+\left[E_{1} \exp (-i K x)+\text { c.c. }\right]
$$

$$
\begin{aligned}
\frac{\partial n_{i 1}}{\partial t} & =-n_{i 1}\left(\omega_{i}+D_{i} K^{2}+i \mu_{i} K E_{0}\right)-N_{d 1}{ }^{+} \omega_{i}, \\
E_{1} & =\frac{i e}{\epsilon K}\left(N_{d 1}{ }^{+}+n_{i 1}\right),
\end{aligned}
$$

where

$$
\omega_{e} \equiv \frac{e \mu_{e} n_{e 0}}{\epsilon}=\frac{e \mu_{e}\left(\frac{\sigma}{h \nu} I_{0}+\beta\right)\left(N_{d}-N_{a}\right)}{\epsilon \gamma_{e} N_{a}}
$$

is the dielectric relaxation rate for conduction band electrons and 


$$
\omega_{i} \equiv \frac{e \mu_{i} n_{i 0}}{\epsilon}
$$

is the dielectric relaxation frequency for conducting ions. In what follows we consider the solutions of Eqs. (4)-(6) in each of the phases of Fig. 1.

\section{B. Compensation of the Electronic Space-Charge Field by Ionic Transport (Phase I)}

In phase I a prerecorded electronic $N_{d 1}{ }^{+}$grating is compensated for in the dark by mobile ionic species. Typically this takes place at an elevated temperature where (e.g., in $\mathrm{LiNbO}_{3}$ ) the ionic conductivity is substantially larger than the electronic conduction associated with thermal detrapping. Therefore the $\mathrm{N}_{d 1}{ }^{+}$modulation can be assumed essentially constant throughout this stage. The space-charge field decays to its equilibrium value as given by

$$
\begin{aligned}
E_{1}^{(\mathrm{I})}(t)= & E_{1}^{(0)}(0)\left\{\frac{D_{i} K^{2}+i K \mu_{i} E_{0}}{\omega_{i}+D_{i} K^{2}+i K \mu_{i} E_{0}}\right. \\
& +\frac{\omega_{i}}{\omega_{i}+D_{i} K^{2}+i K \mu_{i} E_{0}} \\
& \left.\times \exp \left[-\left(\omega_{i}+D_{i} K^{2},+i K \mu_{i} E_{0}\right) t\right]\right\},
\end{aligned}
$$

where $E_{1}^{(0)}(0)$ is the space-charge field of the initially recorded (phase 0 in Fig. 1) electronic grating. The relaxation time constant that describes this phase is

$$
\tau_{\mathrm{I}}=\left(\omega_{i}+D_{i} K^{2}+i K \mu_{i} E_{0}\right)^{-1} .
$$

Because of the diffusion of ions and their finite density the ionic compensation of the electronic space charge is never complete; i.e., there remains, in the steady state, a residual stabilized electric field $E_{1}^{(1)}$. Equation (9) predicts that at the end of phase (I) the value of this steadystate stabilized field is

$$
E_{1}^{(1)}=\frac{D_{i} K^{2}+i K \mu_{i} E_{0}}{\omega_{i}+D_{i} K^{2}+i K \mu_{i} E_{0}} E_{1}^{(0)} .
$$

In data storage applications the aim of this stage is to culminate in as perfect a compensation as possible, i.e., $n_{i 1}\left(t_{1}\right) \approx-N_{d 1}{ }^{+}\left(t_{0}\right)$ or equivalently $E_{1}^{(1)} \ll E_{1}^{(0)}$. For the most practical case of zero applied field this requires, according to Eq. (11), that $\omega_{i} \gg D_{i} K^{2}$ or, equivalently, that

$$
n_{i 0} \gg \frac{\epsilon k_{b} T K^{2}}{e^{2}} .
$$

This condition sets the lower boundary for the optimized density of the free ions in a crystal used for holographic data storage. Further increase of $n_{i 0}$ does not improve the fixing efficiency but decreases the possible storage time because the ionic relaxation frequency $\omega_{i}$ is proportional to $n_{i 0}$ at any temperature. The minimal density of free ions needed for perfect compensation in $\mathrm{LiNbO}_{3}$ is thus $n_{i 0} \gg 5 \times 10^{16} \mathrm{~cm}^{-3}$ (for grating spacing $2 \pi / K=1$ $\mu \mathrm{m})$. Typical $\mathrm{H}^{+}$impurity density in these crystals ${ }^{16}$ is $\sim 10^{18} \mathrm{~cm}^{-3}$, and therefore the ionic compensation is al- ways strong, even if we assume that the only ionic species responsible for hologram fixing is hydrogen.

The above results apply in their basic features also to the case in which the recording phase (0) and the ionic compensation phase (I) are simultaneous. If the ionic compensation takes place in the presence of light under open-circuit conditions, then $E_{0}=-E_{\text {p.v }}$ $=-\kappa \gamma_{e} N_{a} / e \mu_{e}$, where p.v. is the photovoltaic field.

\section{Electronic Decay in the Dark (Phase II)}

Phase II consists of a slow decay in the dark of the ionically compensated electronic grating, the end product of phase I. This decay is due to thermally excited electrons, which proceed to drift and diffuse, thus reducing the strength of hologram $E_{1}^{(1)}$. The ionic grating adiabatically follows the decaying electronic hologram because of the much faster (at elevated temperature ${ }^{4}$ ) adjustment by transport of ions, so at any moment the dynamic equilibrium between the ionic and the electronic gratings is preserved:

$$
\frac{n_{i 1}}{N_{d 1}{ }^{+}}=-\frac{\omega_{i}}{\omega_{i}+D_{i} K^{2}}
$$

Equation (13) follows from Eq. (5) when we take $\partial n_{i 1} / \partial t$ $=0$ and assume a zero dc field $\left(E_{0}=0\right)$. By substituting Eq. (13) into Eqs. (4) and (6) we find that the total space-charge field (i.e., grating amplitude) decays as

$$
\begin{array}{r}
E_{1}^{(\mathrm{II})}(t)=\frac{i e}{\epsilon K}\left[N_{d 1}{ }^{+}(t)+n_{i 1}(t)\right]=E_{1}^{(0)} \frac{D_{i} K^{2}}{\omega_{i}+D_{i} K^{2}} \\
\quad \times \exp \left[-\omega_{e}\left(\frac{D_{i} K^{2}}{D_{i} K^{2}+\omega_{i}}+K^{2} d^{2}\right) t\right], \\
\omega_{e}=\frac{e \mu_{e} \beta\left(N_{d}-N_{a}\right)}{\epsilon \gamma_{e} N_{a}}, \quad d^{2} \equiv \frac{N_{d} k T \epsilon}{N_{a}\left(N_{d}-N_{a}\right) e^{2}}
\end{array}
$$

where $d$ is the electronic Debye screening length that is due to ionized donors. The decay rate

$$
\omega_{\text {decay }}^{\text {(II) }}=\omega_{e}\left(\frac{D_{i} K^{2}}{\omega_{i}+D_{i} K^{2}}+K^{2} d^{2}\right)
$$

is proportional to the electronic dielectric relaxation frequency $\omega_{e}$ (at $I_{0}=0$ ) multiplied by the ionic screening factor plus a term that is due to diffusion of electrons. The electronic decay in the dark, therefore, is slowed down in the presence of large ionic conductivity. The residual electronic dark decay is due primarily to the diffusion [term proportional to $K^{2} d^{2}$ in Eq. (16)] rather than to drift in the hologram's electric field, because the latter is strongly reduced as a result of the conducting ions [see Eqs. (11) and (14)]. Furthermore, in the usual case of strong ionic compensation the dark decay rate is always proportional to the square of the spatial frequency $K$ without saturation at small $K$. This is different from the unscreened case when no ions are present and $\omega_{\text {decay }} \rightarrow \omega_{e}$ for sufficiently small $K, K^{2} d^{2} \ll 1$. The above results are derived for the typical case of zero ap- 
plied field. If the electronic dark decay takes place under an externally applied field $E_{0}$, Eqs. (14)-(16) are modified as follows:

$$
\begin{aligned}
\frac{n_{i 1}}{N_{d 1}{ }^{+}}= & -\frac{\omega_{i}}{\omega_{i}+D_{i} K^{2}+i K \mu_{i} E_{0}} \\
E_{1}^{(\mathrm{II})}(t)= & E_{1}^{(0)} \frac{D_{i} K^{2}+i K \mu_{i} E_{0}}{\omega_{i}+D_{i} K^{2}+i K \mu_{i} E_{0}} \\
& \times \exp \left[-\omega_{e}\left(\frac{D_{i} K^{2}+i K \mu_{i} E_{0}}{\omega_{i}+D_{i} K^{2}+i K \mu_{i} E_{0}}+K^{2} d^{2}\right.\right. \\
& \left.\left.+i K \mu_{e} E_{0}\right) t\right], \\
\omega_{\text {decay }}^{(\mathrm{II})}= & \omega_{e}\left(\frac{D_{i} K^{2}+i K \mu_{i} E_{0}}{\omega_{i}+D_{i} K^{2}+i K \mu_{i} E_{0}}+K^{2} d^{2}\right. \\
& \left.+i K \mu_{e} E_{0}\right) .
\end{aligned}
$$

Experimental data reproduced in Fig. 2 show the dark decay of holographic gratings recorded in $\mathrm{Fe}: \mathrm{LiNbO}_{3}$ at elevated temperature. Two distinct stages of the process (fast and slow) can be identified as fast ionic compensation (phase I) and the much slower thermal decay of the electronic grating screened by the mobile ions (phase II). ${ }^{9}$

\section{Developing or Readout (Phase III)}

Phase III consists of the developing (revealing) of the compensated grating by continuous illumination $\left(I_{0}\right.$ $\neq 0$ ), typically at or near room temperature. This phase also describes what happens when we read the hologram. This causes an increase in the electronic conductivity so that it greatly exceeds that of the ions $\left(\sigma_{e} \gg \sigma_{i}\right)$. The mobile electrons diffuse and drift in the electric field induced by semipermanent ionic charge distribution.

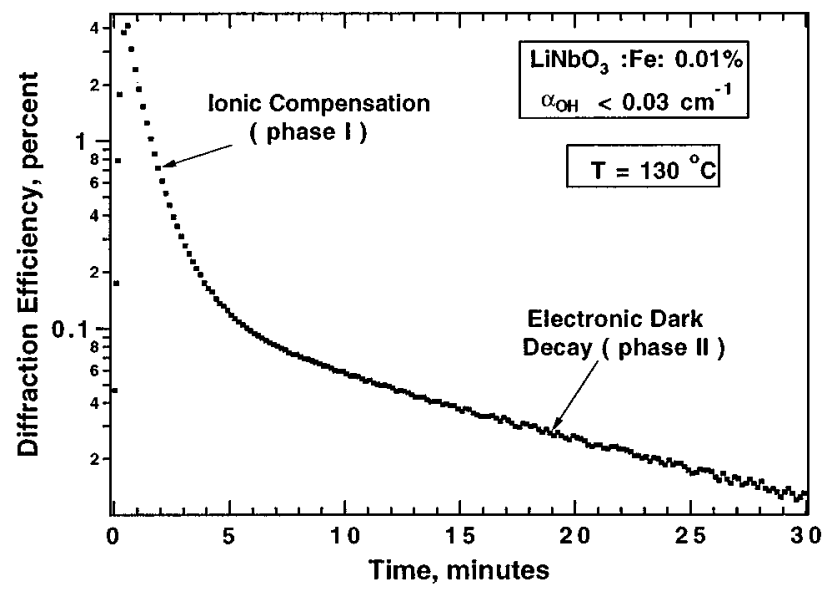

Fig. 2. Diffraction efficiency versus time for a hologram recorded and stored in iron-doped $\mathrm{LiNbO}_{3}$ (partially reduced) at $130{ }^{\circ} \mathrm{C}$. The grating spacing is $2 \pi / K=1.15 \mu \mathrm{m}$. Two stages of the dark decay can be identified as ionic compensation (fast stage of the decay, phase I) and a much slower decay owing to conduction by the thermally excited electrons (phase II).
Therefore, even under spatially homogeneous illumination $I_{0}$, the equilibrium trapped electrons' distribution $\mathrm{N}_{d 1}{ }^{+}$is not uniform but rather screens the fixed ionic pattern. 9,10 The degree of this electronic screening is greatly dependent on the amount of available traps $N_{e}$, the grating spatial frequency $K$, and the strength of the photovoltaic effect. ${ }^{9}$ The inclusion of the photovoltaic effect in this stage makes it necessary to distinguish two different types of boundary condition imposed on the electron current density and the total electric space-charge field. Besides, because the electronic conductivity is dominant in this phase we can consider the ions stationary and derive the expressions for the transient hologram electric field on light readout.

\section{Short Circuit}

Under the short-circuit conditions, $E_{0}=0$, we allow the full dc photovoltaic current $\kappa \alpha I_{0}\left(N_{d}-N_{a}\right)$ to flow. The resulting space-charge holographic field during phase III is

$$
\begin{aligned}
E_{1}^{(\mathrm{III})}(t)= & \frac{i e}{\epsilon K}\left\{\frac{E_{d}-i E_{\mathrm{p} . \mathrm{v} .} N_{a} / N_{d}}{E_{d}+E_{q}-i E_{\mathrm{p} . \mathrm{v} .} N_{a} / N_{d}} n_{i 1}\left(t_{1}\right)\right. \\
& +\left[N_{d 1}^{+}\left(t_{1}\right)\right. \\
& \left.+\frac{E_{q}}{E_{d}+E_{q}-i E_{\mathrm{p} . \mathrm{v} .} N_{a} / N_{d}} n_{i 1}\left(t_{1}\right)\right] \\
& \left.\times \exp \left(-\omega_{1} t\right)\right\} .
\end{aligned}
$$

The relaxation rate that describes this stage of the hologram's life cycle is

$$
\omega_{1} \equiv \omega_{e}\left[1+K^{2} d^{2}-i \frac{E_{\text {p.v. }}}{E_{q}}\left(\frac{N_{a}}{N_{d}}\right)\right],
$$

where the characteristic electric fields are defined as follows:

Characteristic photovoltaic field

$$
E_{\text {p.v. }} \equiv \frac{\kappa \sigma / h \nu I_{0}\left(N_{d}-N_{a}\right)}{e \mu_{e} n_{e 0}}=\frac{\kappa \gamma_{e} N_{a}}{e \mu_{e}},
$$

Limiting space-charge field

$$
E_{q} \equiv \frac{e N_{a}\left(1-N_{a} / N_{d}\right)}{\epsilon K}
$$

Diffusion field

$$
E_{d} \equiv \frac{D_{e} K}{\mu_{e}}=\frac{K k_{b} T}{e} .
$$

Equation (20) predicts a quasi-stabilized field at the end of phase III in the presence of illumination whose value is

$$
E_{1}^{(2)}=\frac{i e}{\epsilon K} n_{i 1}\left(t_{1}\right) \frac{E_{d}-i E_{\text {p.v. }} N_{a} / N_{d}}{E_{d}+E_{q}-i E_{\text {p.v. }} N_{a} / N_{d}}
$$

independently of the initial trapped charge grating $N_{d 1}{ }^{+}\left(t_{1}\right)$. This field can approach the original field $E_{1}^{(0)}$ provided that 


$$
n_{i 1}\left(t_{1}\right) \approx-N_{d 1}^{+}\left(t_{0}\right), \quad E_{1}^{(2)} \approx \frac{i e}{\epsilon K} n_{i 1}\left(t_{1}\right) .
$$

The first of conditions (26) is satisfied when the ionic compensation in phase I is nearly complete, which according to Eq. (11) happens when $\omega_{i} \gg D_{i} K^{2}$; i.e., the density of ions is sufficiently large, $n_{i 0} \gg\left(\epsilon k T / e^{2}\right) K^{2}$ [inequality (12)]. This condition is, in fact, most usually satisfied because of the large density of conducting ions at elevated temperature. The second condition is practically more important and requires that the screening of the fixed ionic space charge by trapped electrons during the readout not be strong. According to Eq. (25) this is the case, provided that

$$
\left|E_{d}-i\left(N_{a} / N_{d}\right) E_{\text {p.v. }}\right| \gg E_{q}
$$

This condition can be satisfied for high spatial frequencies, namely, $K \gg K_{e} \equiv 1 / d$ or, in the case of a strong photovoltaic effect, $E_{\text {p.v. }} \gg E_{q}$. In iron-doped $\mathrm{LiNbO}_{3}$, high developing efficiency can be achieved only in strongly oxidized (large photovoltaic field) weakly $(<0.05$ wt. \%) iron-doped crystals because the limiting spacecharge field $E_{q}$ largely exceeds the photovoltaic and diffusion fields otherwise.

\section{Open Circuit}

In the open-circuit case no spatial dc current flows through the crystal, but there exists an internally generated (because of the photovoltaic effect) dc field $E_{0}$ in the crystal. The solution for the spatially varying component (i.e., hologram) of the electric space-charge field is

$$
\begin{aligned}
E_{1}^{(\mathrm{III})}(t)= & \frac{i e}{\epsilon K}\left\{\frac{E_{d}+i E_{\text {p.v. }}\left(N_{d}-N_{a}\right) / N_{d}}{E_{d}+E_{q}+i E_{\text {p.v. }}\left(N_{d}-N_{a}\right) / N_{d}} n_{i 1}\left(t_{1}\right)\right. \\
& +\left[N_{d 1}^{+}\left(t_{1}\right)\right. \\
& \left.+\frac{E_{q}}{E_{d}+E_{q}+i E_{\text {p.v. }}\left(N_{d}-N_{a}\right) / N_{d}} n_{i 1}\left(t_{1}\right)\right] \\
& \left.\times \exp \left(-\omega_{1} t\right)\right\} .
\end{aligned}
$$

The characteristic relaxation rate in this case is

$$
\omega_{1}=\omega_{e}\left[1+K^{2} d^{2}+i \frac{E_{\text {p.v. }}}{E_{q}} \frac{\left(N_{d}-N_{a}\right)}{N_{d}}\right] .
$$

In $\mathrm{LiNbO}_{3}$ the open-circuit case, in general, gives lower diffraction efficiencies ${ }^{17}$ during the initial recording; hence we expect a weaker initial hologram magnitude [smaller $E_{1}^{(0)}$ ]. Besides, because in strongly oxidized $\mathrm{LiNbO}_{3} N_{d}-N_{a} \ll N_{d}$, the unscreening of the ionic hologram that is due to the photovoltaic effect is also reduced, leading to even more-weakly developed holograms than in the short-circuited case [compare Eqs. (20) and (28)].

\section{E. Ionic Decay on Readout (Phase IV)}

1. Short Circuit

Phase IV relates directly to the lifetime of the hologram in actual applications. It involves the decay of the ionic backbone grating that is due to ion (drift+diffusive) transport. In phase III we neglected ionic transport and considered the transient behavior of the grating before the dynamic equilibrium is reached. This decision was justified because the duration of that phase is short compared with the ionic decay time $\left(\omega_{i}+D_{i} K^{2}\right)^{-1}$. The dynamic electronic compensation resulted in a stabilized space-charge field (hologram) as given by Eq. (25). This field proceeds to decay to zero because of the ionic transport that erases the ionic backbone charge $n_{i 1}$. This phase usually takes place under light illumination, when the electronic photoconductivity is much larger than the ionic conductivity. The key physical assumption here is that the ionic decay process is sufficiently slow that, while it takes place, the faster electronic transport in the presence of light causes the trapped charge density $N_{d 1}{ }^{+}(t)$ always to be in equilibrium with $n_{i 1}$. This equilibrium ratio is obtained from Eqs. (6) and (25) for $t \rightarrow \infty$ (i.e., after the relaxation of the transient):

$$
\frac{N_{d 1}{ }^{+}(t)}{n_{i 1}(t)}=-\frac{E_{q}}{E_{d}+E_{q}-i E_{\text {p.v. }} N_{a} / N_{d}} .
$$

With this last condition we obtain from Eq. (5)

$$
\begin{aligned}
n_{i 1}^{(\mathrm{IV})}(t)= & n_{i 1}\left(t_{2}\right) \exp \left\{-\left[\omega_{i}\left(\frac{E_{d}-i E_{\text {p.v. }} N_{a} / N_{d}}{E_{d}+E_{q}-i E_{\text {p.v. }} N_{a} / N_{d}}\right)\right.\right. \\
& \left.\left.+D_{i} K^{2}\right] t\right\}
\end{aligned}
$$

for the short-circuit case. We recall that $n_{i 1}\left(t_{2}\right)$ $\approx n_{i 1}\left(t_{1}\right)$ because phase III is too short for significant ionic transport.

The corresponding grating fields are obtained from Eqs. (6) and (30) and by replacing $n_{i 1}(t)$ by its solution, Eq. (31). The result is that during phase IV the electronically screened ionic hologram decays to zero because of ionic transport, which, however, is slowed down because the partial electronic compensation

$$
\begin{aligned}
E_{1}^{(\mathrm{IV})}(t)= & \frac{i e}{\epsilon K}\left(\frac{E_{d}-i E_{\mathrm{p} . \mathrm{v} .} N_{a} / N_{d}}{E_{d}+E_{q}-i E_{\mathrm{p} . \mathrm{v} .} N_{a} / N_{d}}\right) n_{i 1}\left(t_{1}\right) \\
& \times \exp \left\{-\left[\omega_{i}\left(\frac{E_{d}-i E_{\mathrm{p} . \mathrm{v} .} N_{a} / N_{d}}{E_{d}+E_{q}-i E_{\mathrm{p} . \mathrm{v} .} N_{a} / N_{d}}\right)\right.\right. \\
& \left.\left.+D_{i} K^{2}\right] t\right\} .
\end{aligned}
$$

\section{Open Circuit}

The decay dynamics of the ionic backbone grating in the open-circuit case can be found by use of a similar procedure. The equilibrium trap density modulation in this case is

$$
\frac{N_{d 1}{ }^{+}(t)}{n_{i 1}(t)}=-\frac{E_{q}}{E_{d}+E_{q}+i E_{\mathrm{p} . \mathrm{v} .}\left(N_{d}-N_{a}\right) / N_{d}},
$$

and the total hologram space-charge field of electronically compensated ionic grating decay is given by 
$E_{1}^{(\mathrm{IV})}(t)$

$$
\begin{aligned}
= & \frac{i e}{\epsilon K}\left[\frac{E_{d}+i E_{\text {p.v. }}\left(N_{d}-N_{a}\right) / N_{d}}{E_{d}+E_{q}+i E_{\text {p.v. }}\left(N_{d}-N_{a}\right) / N_{d}}\right] n_{i 1}\left(t_{1}\right) \\
& \times \exp \left(-\left\{\omega_{i}\left[\frac{E_{d}+i E_{\text {p.v. }}\left(N_{d}-N_{a}\right) / N_{d}}{E_{d}+E_{q}+i E_{\text {p.v. }}\left(N_{d}-N_{a}\right) / N_{d}}\right]\right.\right. \\
& \left.\left.+D_{i} K^{2}\right\} t\right) .
\end{aligned}
$$

\section{EXPERIMENTS}

\section{A. General Remarks on Hologram Fixing and Ionic Conduction in $\mathrm{LiNbO}_{3}$}

The hologram thermal ionic fixing in photorefractive $\mathrm{LiNbO}_{3}$ has been studied extensively. ${ }^{4,9,11-14,18-22}$ In general, it is agreed that above $70-80{ }^{\circ} \mathrm{C}$ the ionic conductivity in this crystal largely prevails over the dark electronic conduction because of $\mathrm{Fe}^{2+}$-electron detrapping. At temperatures somewhat below $60{ }^{\circ} \mathrm{C}$, however, the dark conductivity is due predominantly to electrons and is characterized by a small activation energy $(0.1-0.4 \mathrm{eV})$ that is due to the thermally assisted small polaron electronic conduction associated with the $\mathrm{Nb}_{\mathrm{Li}}{ }^{4+}$ defect center. $^{23}$ This defect (shallow electron trap) is due to the inherent lithium deficiency in congruent $\mathrm{LiNbO}_{3}$, and it plays an important role in considerations of crystal dark conductivity at and near room temperature. In the presence of light, however, the photoconductivity is governed by iron impurities in iron-doped $\mathrm{LiNbO}_{3} \cdot{ }^{24}$

The thermally activated ionic (and, similarly, the thermally activated electronic) conductivity obeys an Arrhenius-type dependence on the absolute temperature $T$ :

$$
\sigma_{i}=e n_{i} \mu_{0} \exp \left(-E_{a} / k_{b} T\right),
$$

where $n_{i}$ is the density of ions and $E_{a}$ is the activation energy. At any given temperature the lifetime of the ionic space charge is determined by the residual ionic conductivity given by Eqs. (8), (32), and (35) and, importantly, is inversely proportional to the density of the conducting ions. It is well established ${ }^{19,25}$ that the ionic conductivity in as-grown and hydrogen-doped $\mathrm{LiNbO}_{3}$ crystals is predominantly due to the $\mathrm{H}^{+}$ions. Hydrogen normally is located in the oxygen planes along the $\mathrm{O}-\mathrm{O}$ bond, and its relative contents can be evaluated therefore as the strength (peak or integral) of the $\mathrm{OH}^{-}$stretching vibration absorption line near $2.87 \mu \mathrm{m} .^{26}$ Vormann et $a l .{ }^{19}$ showed unambiguously that in as-grown and $\mathrm{H}^{+}$-doped crystals the ionic conductivity is due to hydrogen and deduced the activation energy of $E_{a}=1.2 \mathrm{eV}$ for its migration. It was recognized, however, that in asgrown $\mathrm{LiNbO}_{3}$ the residual ionic conductivity gives an extrapolated lifetime of an ionic hologram of only 50-70 days at room temperature, and therefore increasing the available storage time requires a substantial reduction of ion density. ${ }^{9,27}$ The increase is achieved in our research by substantial dehydration of as-grown crystals.

In what follows, we describe our experimental study of the long-lifetime hologram fixing and ionic conductivity in iron-doped congruent and noncongruent $\mathrm{LiNbO}_{3}$ and also present some results on high- and low-temperature electronic dark conductivity.

\section{B. Experimental Procedure}

We investigated hologram fixing and the temperature dependence of the ionic conductivity in iron-doped $\mathrm{LiNbO}_{3}$ crystals grown by Deltronic Crystal Industries. An $\mathrm{Ar}^{+}$-ion laser beam with wavelength $\lambda=488 \mathrm{~nm}$ was used in most of the experiments. The crystal was placed on a heater plate whose temperature was controlled within $0.1^{\circ} \mathrm{C}$ accuracy. The crystal and the heater were placed in a vacuum chamber to avoid the optical phase perturbations during the recording stage that otherwise occur because of air density fluctuations in the vicinity of the hot plate. The samples measured $5 \mathrm{~mm} \times 5 \mathrm{~mm}$ $\times 10 \mathrm{~mm}$, with the longest edge lying along the optical $c$ axis, except for the vapor-transport-equilibrated (VTE) sample, which was only $\sim 1 \mathrm{~mm}$ thick. The crystals were short circuited by conducting electrodes placed over the four facets of the crystal.

The holograms were recorded with two equal-intensity ordinarily polarized $488-\mathrm{nm}$ beams with the grating vector lying along the $c$-axis direction. The recording beams were expanded to approximately $1 \mathrm{~cm}$ in diameter and covered the whole crystal during the recording. The total recording (and erasing) optical intensity was $\sim 100$ $\mathrm{mW} / \mathrm{cm}^{2}$. The holographic diffraction efficiency was monitored with a weak Bragg-matched extraordinarily polarized $\mathrm{He}-\mathrm{Ne}$ laser beam. The diffraction efficiency was sampled at a controllable rate (determined mostly by the temperature of the sample during the experiment) to minimize the erasure of the hologram by the probing beam itself. During the developing stage a non-Braggmatched 488-nm expanded beam was used to issue an approximate uniformity of the erasing intensity throughout the volume of the sample. After each experiment the crystal was heated to $\sim 230{ }^{\circ} \mathrm{C}$ and soaked for $\sim 30 \mathrm{~min}$ to ensure uniform distributions of both electrons and ions.

In the fixing experiments (low-high-low fixing; Subsection 3.C) the grating was recorded at a low temperature $\left(50^{\circ} \mathrm{C}\right)$. The sample was heated in the dark to cause fast ionic transport and then cooled to the temperature of recording $\left(50{ }^{\circ} \mathrm{C}\right)$. The resulting electronically compensated ionic grating was revealed (developed) with a non-Bragg-matched erasing beam.

The dark decay of an ionically compensated trapped electron grating is measured as the decay rate of the weak hologram (see Fig. 2, phase 2) left behind after the ionic compensation is complete. Because of the fast ionic screening and the high ion density, a prolonged (5-30$\mathrm{min}$ ) recording of the grating at an elevated temperature $\left(T>130{ }^{\circ} \mathrm{C}\right)$ was required for a measurable diffracted signal to be obtained during this stage.

We determined the ionic hologram lifetime (Subsection 3.F) by monitoring the dark decay (Fig. 2; phase 1) of gratings recorded and stored at different (although constant throughout each measurement) temperatures. The recording time was kept at a minimum (until the holographic diffraction efficiency reached 10-20\%). 


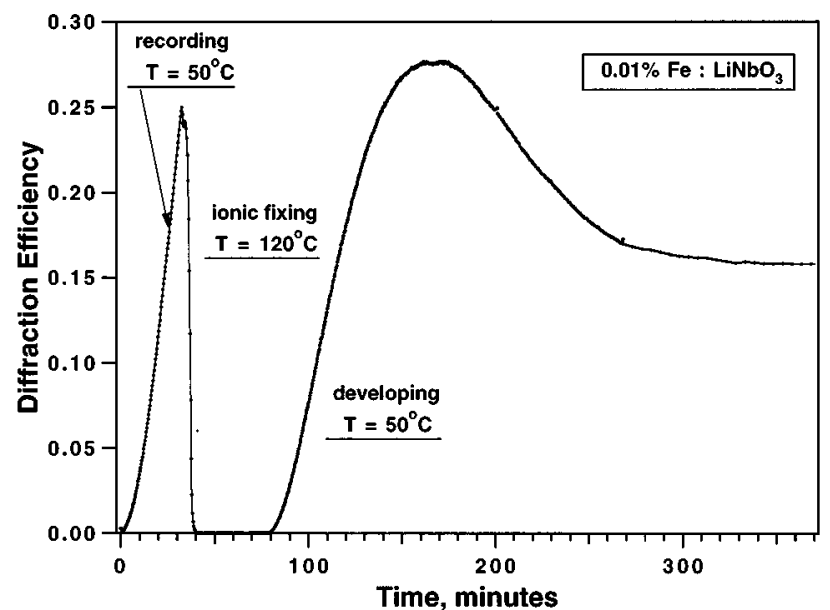

(a)

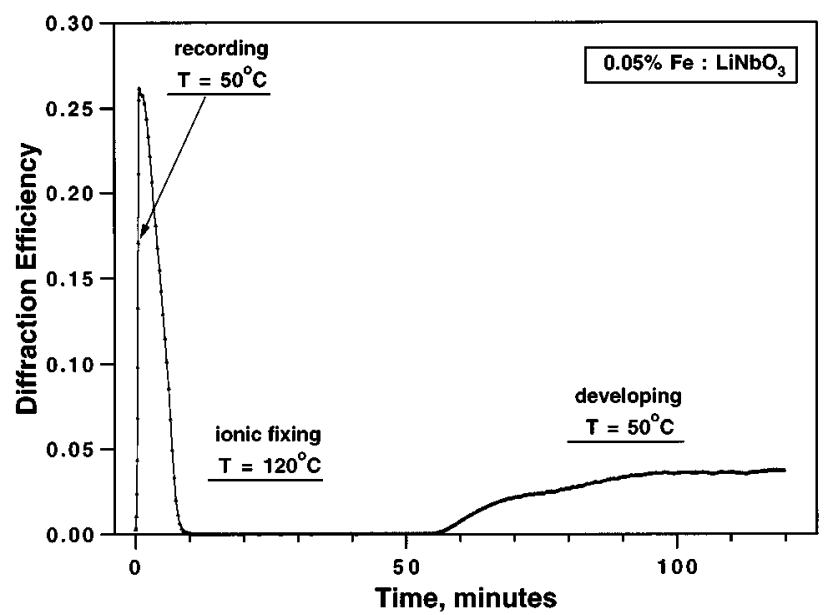

(b)

Fig. 3. Ionic fixing in oxidized dehydrated $\mathrm{LiNbO}_{3}$ crystals with the percentages of iron doping indicated. Initial diffraction efficiencies are $\sim 25 \%$, and the grating spacing is $2 \pi / K \approx 1 \mu \mathrm{m}$ in both cases.

\section{Hologram Low-High-Low Fixing}

High diffraction efficiencies of the fixed (reflection) holograms can be obtained by prolonged recording at high temperatures. $^{28}$ In such a case, effectively the hologram is recorded and ionically compensated many times, leading to a substantially higher ionic space-charge perturbation. However, this procedure is not practical for a complex image-bearing hologram because of the inevitable degradation of the Bragg conditions as the result of thermal mismatch. Only simple single gratings can be recorded and fixed by this method.

In holographic data storage it is essential that recording and reconstruction of (fixed) holograms be performed at the same temperature. We therefore investigated the fixing of holograms by a temperature cycling method (Figs. 3 and 4). In all the experiments on low-high-low fixing the holograms were initially recorded at $50{ }^{\circ} \mathrm{C}$. The ionic compensation is slow at this temperature, and the dark decay time (exponential) of the holograms was more than $100 \mathrm{~h}$ in the samples used. After the recording the writing beams were blocked and the sample with the hologram was heated to $\sim 120{ }^{\circ} \mathrm{C}$ to cause fast ionic transport. Once the hologram was compensated, the temperature was returned to the initial value at the recording stage. When the sample temperature was stabilized at $50{ }^{\circ} \mathrm{C}$ a non-Bragg-matched expanded beam was shone directly onto the crystal to develop (reveal) the fixed holograms. Both crystals used in this experiment had a low hydrogen-impurity content (see Subsection 2.6 below); the magnitude of the $\mathrm{OH}^{-}$stretching absorption band was $<0.01 \mathrm{~cm}^{-1}$ (i.e., below the resolution limit of the spectrometer) and $0.035 \mathrm{~cm}^{-1}$ in $0.05 \%$ and $0.01 \%$ iron-doped samples, respectively.

In the experiments shown in Fig. 3 the holograms were recorded for short periods of time until they reached the same magnitude $(\sim 25 \%)$. This low value of diffraction efficiency was chosen to avoid the saturation effects of the $\sin ^{2}$ dependence of diffraction efficiency on the index perturbation and thus to permit direct comparison of the relative fixing and development efficiencies. In agree-

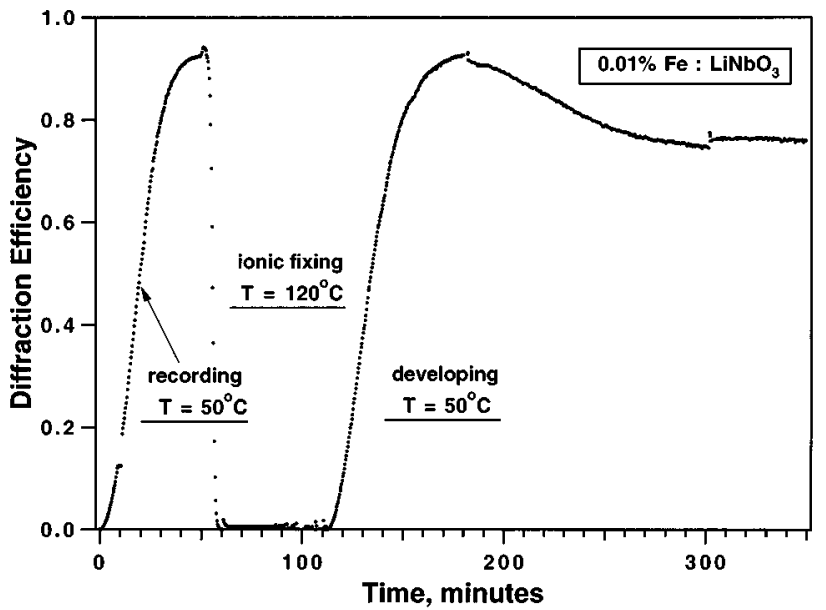

(a)

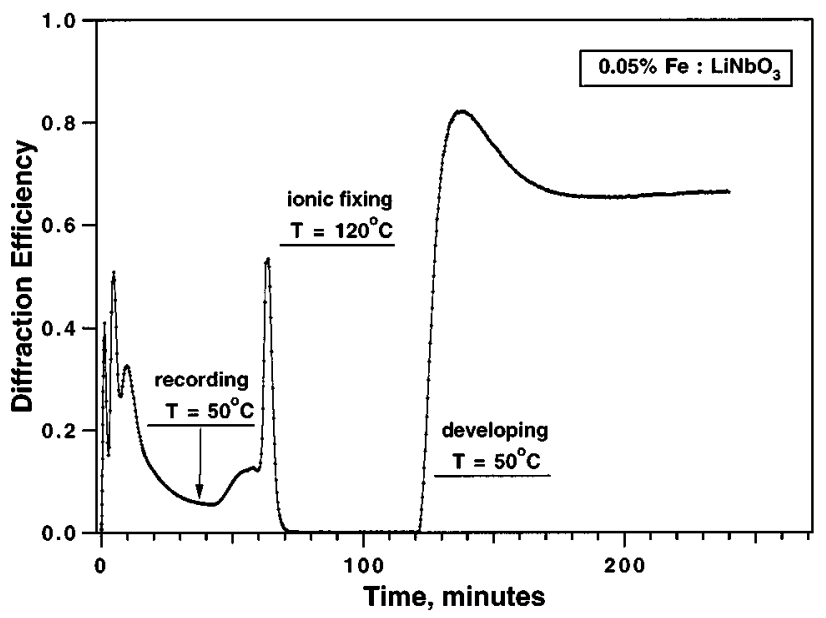

(b)

Fig. 4. Ionic fixing in oxidized dehydrated $\mathrm{LiNbO}_{3}$ crystals with the percentages of iron doping indicated. The holograms were recorded for $\sim 1 \mathrm{~h}$ until the saturation was reached. The oscillations in the recording curve in (b) are due to the $\sin ^{2}$ dependence of $\eta$ on index perturbation $\delta n$ and to beam coupling of recording beams. The grating spacing is $2 \pi / K \approx 1 \mu \mathrm{m}$ in both cases. 
ment with theoretical predictions (Subsection 2.D) the fixing and development efficiency depends strongly on iron doping and the oxidation state of the crystal. In irondoped $\mathrm{LiNbO}_{3}$ the limiting space-charge field $E_{q}$ is proportional to the $\mathrm{Fe}^{2+}$ density [see Eq. (23)]:

$$
E_{q}=\frac{e\left[\mathrm{Fe}^{3+}\right]\left[\mathrm{Fe}^{2+}\right]}{\epsilon K\left(\left[\mathrm{Fe}^{3+}\right]+\left[\mathrm{Fe}^{2+}\right]\right)} \approx \frac{e\left[\mathrm{Fe}^{2+}\right]}{\epsilon K} .
$$

The limiting space-charge field $E_{q}$ determines the degree of electronic screening of a fixed ionic hologram [Eq. (25)], and the fixing and developing efficiency is decreased with increasing $\left[\mathrm{Fe}^{2+}\right.$ ] [Fig. 3(b)]. At the same time, almost $70 \%$ reconstruction efficiency (i.e., weak screening) is realizable in weakly iron-doped crystals, as shown in Fig. 3(a). This observation also demonstrates that the electronic detrapping from $\mathrm{Fe}^{2+}$ sites can be avoided even in $\mathrm{LiNbO}_{3}$ with largely reduced ionic conductivity (i.e., low hydrogen-impurity content).

Figure 4 shows the fixing experiments in which the recording was performed until the perturbation in the index reached its steady state. The maximal index perturbation that can be induced in oxidized $\mathrm{LiNbO}_{3}$ is larger the higher the iron doping. However, the developing efficiency decreases with iron doping as well. The net result is that the fixed holograms have almost the same efficiencies in the crystals with different iron-dopant concentrations (Fig. 4) when the low-high-low fixing technique is used.

\section{Dark Electronic Decay}

A hologram recorded at elevated temperature and left in the dark experiences fast ionic compensation (Fig. 2, phase 1), which, of course, also takes place during recording if the ionic conductivity is larger than or comparable with the electronic photoconductivity. Fast ionic decay is followed by a much slower electronic grating decay, which is due to electron detrapping from $\mathrm{Fe}^{2+}$ sites (Fig. 2; phase 2). The ionic compensation is strong because of the relatively high density $\left(>10^{18} \mathrm{~cm}^{-3}\right)$ of compensating ions, and therefore the residual weak index grating may have both electro-optic [that is due to the remaining electric field as given by Eq. (11)] and photochromic contributions. $^{29}$ An Arrhenius plot of dark electronic decay time in $0.15 \%$ iron-doped congruent $\mathrm{LiNbO}_{3}$ for different grating spacings is shown in Fig. 5. The corresponding ionic lifetimes are also shown for reference. In agreement with theory, the dark electronic grating lifetime strongly depends on the grating spacing [Eq. (16)]. At the same time, the ionic decay time (fast stage of the decay) is almost independent of the grating spacing. We also observe that, if the erasing light is shone on the crystal during phase 2 of Fig. 2, the weak residual grating decays rapidly to zero at a rate that is close to that of the recording stage. Thus the slow decay can be enhanced by light and therefore is indeed electronic. It is worth emphasizing that above $\sim 90{ }^{\circ} \mathrm{C}$, even in heavily iron-doped oxidized $\mathrm{LiNbO}_{3}$ crystals, the ionic conductivity is higher than the dark electronic conduction (because of thermal detrapping from both $\mathrm{Fe}^{2+}$ sites and the small polaron $\mathrm{Nb}_{\mathrm{Li}}{ }^{4+}$ shallow trap defect).

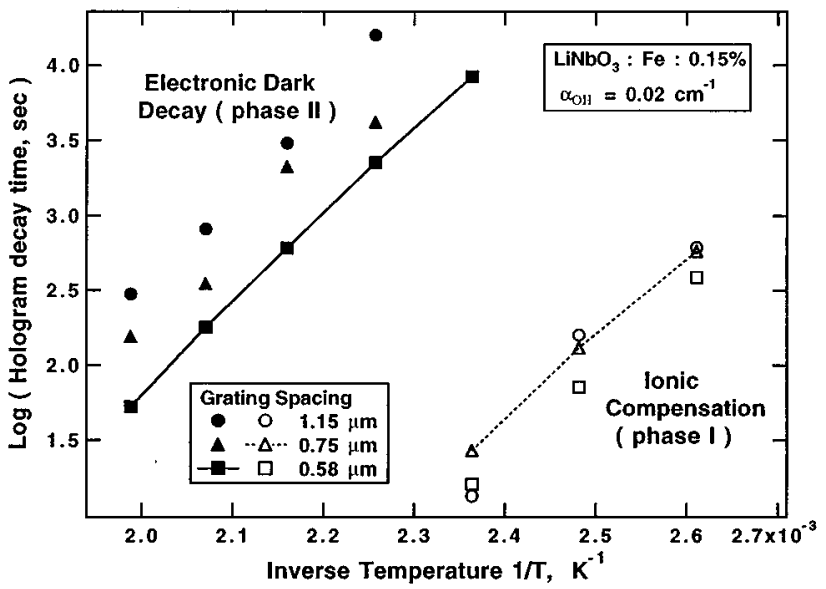

Fig. 5. Arrhenius plot of the hologram's dark electronic decay time. A plot of ionic compensation also shown for comparison.

\section{E. Ionic Hologram Decay on Readout}

Ionic transport is the mechanism that is responsible both for the ionic compensation in the dark (Fig. 1, phase I) and for the decay of the fixed ionic grating during the readout (Fig. 1, phase IV). The latter, however, can be slowed to a certain amount because of the compensation effect of the electronic grating. Figure 6 shows the evolution of two holograms that were recorded, fixed, and reconstructed at $90{ }^{\circ} \mathrm{C}$ (the temperature was kept constant throughout the experiments) in the dehydrated lithium crystals with different iron-dopant concentrations. In the crystal with low iron doping $\left(0.01 \%\right.$ iron, small $\left.E_{q}\right)$ the rate of ionic decay during the readout is close to the dark compensation rate. This is not surprising because the electronic screening is weak in this crystal [Fig. 3(a)]. The higher-doped crystal exhibits substantial electronic screening [Fig. 6(b)], and consequently the decay on development is slower [by a factor of $\sim 5$ in the conditions of Fig. 6(b)] than that during the ionic compensation. At the same time, the reconstruction efficiency (in this case the ratio between the maximal value of the reconstructed signal and the initially recorded signal) is substantially lower than in the case of weak electronic compensation [see also Fig. 3(b)]. It can be concluded, therefore, that for a given residual ionic conductivity and a given storage temperature an increase in the lifetime of an electronically compensated fixed ionic hologram can be achieved only at the proportionate expense of its reconstruction efficiency.

\section{F. Lifetime of a Fixed Ionic Hologram}

The lifetime of a fixed ionic grating is determined mainly by the conductivity $\sigma_{i}$ of ionic species at a given temperature $T$, i.e., $\tau \propto \epsilon \epsilon_{0} / \sigma_{i}$, where $\sigma_{i}$ is given by Eq. (35). To determine the ionic conductivity and the corresponding activation energies we measured the dark decay time (Fig. 2, phase 1) of the holograms recorded and stored at different (although constant throughout each experiment) temperatures. We found that in as-grown congruent $\mathrm{LiNbO}_{3}$ crystals with fairly high hydrogen content (measured as the strength of the $\mathrm{OH}^{-}$absorption near 2.87 $\mu \mathrm{m})$ the ionic conductivity exhibits an $\sim 1.2-\mathrm{eV}$ activation 
energy and is relatively high [Fig. 7, curve (a)]. This activation energy is characteristic of hydrogen conductivity. ${ }^{19,25}$ The lifetime extrapolated to room temperature $\left(24^{\circ} \mathrm{C}\right)$ is $\sim 50$ days, which is too short for the majority of applications. ${ }^{27}$ Partial electronic screening under illumination slows down the ionic decay but at the same time decreases the grating strength [see Subsection 3.E and Eq. (32)]. Therefore the most feasible mechanism for increasing the fixed hologram lifetime is the decrease in the ionic conductivity that one can achieve by reducing the total density of conducting ions. Hightemperature $\left(\sim 950^{\circ} \mathrm{C}\right)$ postgrowth processing in a dry oxygen atmosphere results in a substantial reduction of as much as 100 times compared with as-grown $\mathrm{OH}^{-}$density in the crystal and leads to a significant increase in the ionic hologram's lifetime. However, we found [Fig. 7,

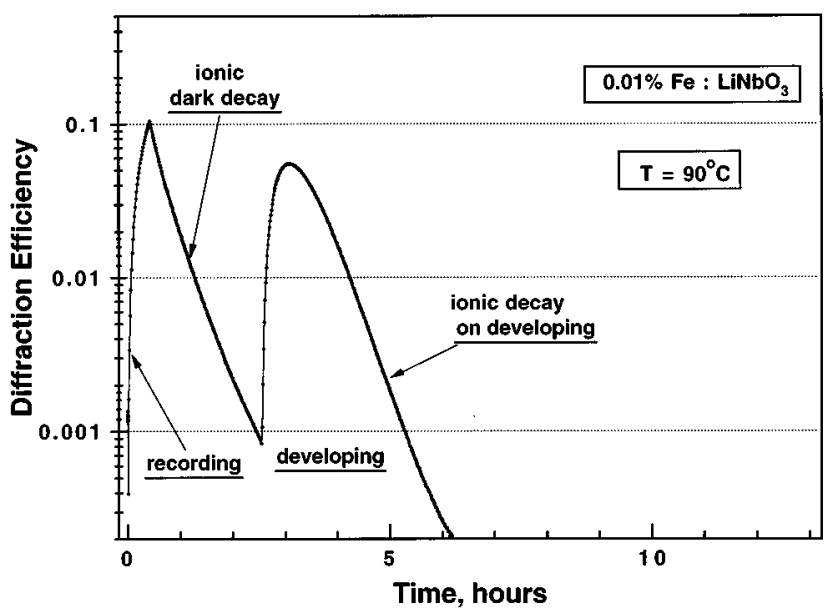

(a)

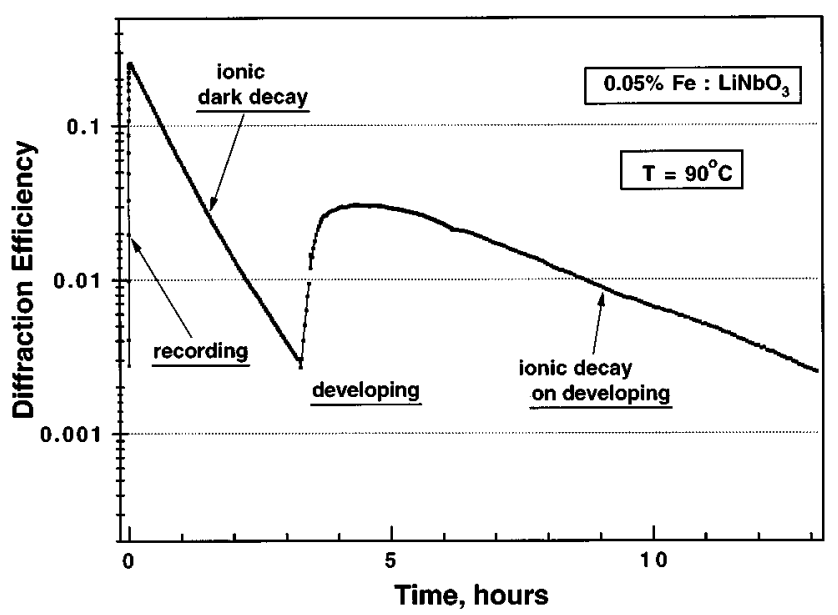

(b)

Fig. 6. Recording, ionic compensation, developing, and final decay of the holograms in oxidized dehydrated $\mathrm{LiNbO}_{3}$ crystals with iron doping as indicated. In (b) the fixed grating decay is $\sim 5$ times slower than the ionic compensation (ionic dark decay) because of stronger electronic screening, whereas in (a) they are almost equal. The ratio between the ionic compensation rate in the dark and the decay rate of the fixed ionic grating on readout is preserved as long as the ionic conductivity is much smaller than the electronic photoconductivity (i.e., for $T \leqslant \sim 100{ }^{\circ} \mathrm{C}$ ), The grating spacing is $2 \pi / K \approx 1 \mu \mathrm{m}$ in both cases.

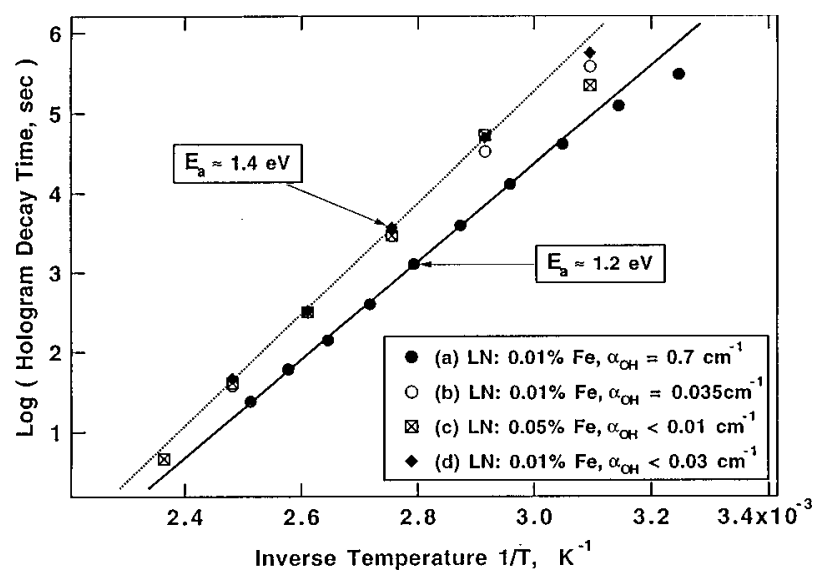

Fig. 7. Arrhenius plot of the ionic hologram lifetime in (a) asgrown ( $E_{a}=1.2 \mathrm{eV}$ ) crystal; (b), (c) samples with low hydrogenimpurity content and different iron doping $\left(E_{a}=1.4 \mathrm{eV}\right) ;(\mathrm{d})$ $\mathrm{Li}_{2} \mathrm{O}$ equilibrated (VTE processed) crystal. The falloff in the hologram lifetime in the low-temperature range (i.e., when $T<70^{\circ} \mathrm{C}$ ) is due to the electronic decay caused by the shallow trap $\left(\mathrm{Nb}_{\mathrm{Li}}{ }^{4+}\right.$ small polaron defect) thermal electronic conduction in nonstoichiometric $\mathrm{LiNbO}_{3}$.

curves (b) and (c)] that, after substantial hydrogen removal, the ionic conductivity is determined by a species other than hydrogen. The ionic conductivity in dehydrated crystals does not depend on the hydrogen density, as opposed to the linear dependence of $\sigma_{i}$ on $\left[\mathrm{OH}^{-}\right]$found in as-grown and hydrogen-doped samples. ${ }^{19,25}$ The activation energy also differs from that typical for the $\mathrm{H}^{+}$-impurity transport and is equal to $\sim 1.4 \mathrm{eV}$. The fixed ionic hologram lifetime in dehydrated samples at room temperature $\left(24^{\circ} \mathrm{C}\right)$ can be extrapolated to be $\sim 2$ years.

To investigate the influence of lithium vacancies on the ionic conductivity ${ }^{30}$ we prepared a more stoichiometric sample, using the VTE technique. ${ }^{31,32}$ The processing temperature was $1080^{\circ} \mathrm{C}$ and the processing time was $400 \mathrm{~h}$ for a 1 -mm-thick $a$-cut $\mathrm{LiNbO}_{3}$ crystal. The $\mathrm{Li}_{2} \mathrm{O}$ content in this sample was estimated to be at least $49.5 \%$ [congruent $\mathrm{LiNbO}_{3}$ contains $48.6 \% \mathrm{Li}_{2} \mathrm{O}$ (Ref. 33)] from phase-matching angle measurements for secondharmonic generation at $1.064 \mu \mathrm{m} .{ }^{34}$ In the temperature range studied in our experiments the ionic conductivity in the VTE sample also exhibits an $\sim 1.4$-eV activation energy [curve (d) of Fig. 7] and is nearly the same as the one in congruent dehydrated $\mathrm{LiNbO}_{3}$. This may rule out the mechanisms of ionic conduction that involve a substantial role of lithium vacancies-lithium deficiency. The nature of ionic conducting species in the dehydrated $\mathrm{LiNbO}_{3}$ crystals has yet to be determined. Migration of lithium self-interstitials ${ }^{32}$ may be a mechanism responsible for the ionic conductivity with an activation energy of $1.4 \mathrm{eV}$.

Note that the ionic conductivity with the 1.4-eV activation energy and the substantially increased ionic hologram lifetimes at room temperature can be found only in fully oxidized $\mathrm{LiNbO}_{3}$ crystals with a very low hydrogenimpurity content $\left(\left[\mathrm{OH}^{-}\right]<5 \times 10^{17} \mathrm{~cm}^{-3}\right.$, that is, for $\mathrm{OH}^{-}$absorption at $2.87 \mu \mathrm{m}$ of less than $0.05 \mathrm{~cm}^{-1}$ ). In samples with higher hydrogen contents the dark decay 
time constant may exhibit activation energies that range widely from 0.9 to $1.4 \mathrm{eV}$, depending on the density of $\mathrm{H}^{+}$ impurity, the range of temperatures used in the experiments, and the oxidation state and density of the iron dopant. $^{20-22,35}$

\section{CONCLUSIONS}

We have presented a theoretical model that describes holographic ionic fixing, readout, and storage dynamics in photorefractive materials. There is good agreement between the experimental results on hologram fixing in iron-doped photorefractive $\mathrm{LiNbO}_{3}$ and the prediction of the theoretical formulation. Experimental study reveals some new features in the holographic ionic fixing and related low-temperature ionic conductivity in $\mathrm{LiNbO}_{3}$. A few main conclusions are worth emphasizing:

(a) At elevated temperatures $\left(T>\sim 60{ }^{\circ} \mathrm{C}\right.$ for $\mathrm{LiNbO}_{3}$ ) the initial decay of the recorded grating is due to ionic compensation (phase I). Under dark storage conditions the (phase II) decay as described by Eq. (14) is due to the slowing of thermal electronic transport by ionic screening.

(b) Nearly perfect development and fixing [i.e., $E_{1}\left(t_{2}\right) \approx E_{1}\left(t_{0}\right)$ in Fig. 1] can be obtained, provided that the screening of the residual ionic grating by electronic grating is small on readout. According to Eq. (25) this happens when $\left|E_{d}-i N_{a} / N_{d} E_{\text {p.v. }}\right| \gg E_{q}$. This condition is realized in weakly iron-doped oxidized $\mathrm{LiNbO}_{3}$ crystals with strong photovoltaic response. The fixing efficiency can be close to $100 \%$ in such crystals.

(c) Under illumination (phase IV) the ionic backbone hologram is quasi-stabilized. The residual decay is due to the transport of the ionic charge [Eq. (32)]. A major reduction of the ionic conductivity at the operating temperature is necessary to bring this decay rate to the commercially acceptable range of lifetimes of $\geqslant 10$ years. An increase of the fixed hologram lifetime can be also achieved as a result of strong electronic compensation but only at the expense of the hologram's reconstruction efficiency [Eq. 32].

(d) A substantial increase in the fixed (ionic hologram) lifetime can be realized in $\mathrm{LiNbO}_{3}$ crystals with low hydrogen contents. The residual ionic conductivity (and associated hologram decay time constant) exhibits an $\sim 1.4-\mathrm{eV}$ activation energy and is not due to protonic conduction. Fixed hologram lifetimes of $\sim 2$ years at room temperature are expected for dehydrated crystals.

\section{ACKNOWLEDGMENTS}

A. Yariv and S. Orlov acknowledge the support of an Advanced Research Projects Agency University Research Initiative Grant (L. N. Durvasula) and of the U.S. Army Research Office (R. Guenther). S. Orlov thanks Tak Cheung (Caltech) for his help with the measurements of the dark electronic decay (Fig. 5). G. Rakuljic acknowledges the support of the U.S. Army Research Office under contract DAAH04-95-C-0063, with funding from the Advanced Research Projects Agency.
Part of this research was conducted while A. Yariv was a consultant to Accuwave Corporation.

\section{REFERENCES}

1. J. F. Heanue, M. C. Bashaw, and L. Hesselink, "Volume holographic storage and retrieval of digital data," Science 265, 749-752 (1994).

2. D. Psaltis and F. Mok, "Holographic memories," Sci. Am. 273, 70-76 (1995)

3. J. H. Hong, I. McMichael, T. Y. Chang, W. Christian, and E. G. Paek, "Volume holographic memory systems: techniques and architectures," Opt. Eng. 34, 2193-2203 (1995).

4. J. J. Amodei and D. L. Staebler, "Holographic pattern fixing in electrooptic crystals," Appl. Phys. Lett. 18, 540-542 (1971).

5. F. Micheron and G. Bismuth, "Electrical control of fixation and erasure of holographic patterns in ferroelectric materials," Appl. Phys. Lett. 20, 79-81 (1972).

6. D. Kirillov and J. Feinberg, "Fixable complementary gratings in photorefractive $\mathrm{BaTiO}_{3}$," Opt. Lett. 16, 1520-1522 (1991).

7. G. Montemezzani and P. Günter, "Thermal hologram fixing in pure and doped $\mathrm{KNbO}_{3}$ crystals," J. Opt. Soc. Am. B 7, 2323-2328 (1990).

8. G. Montemezzani, M. Zgonik, and P. Günter, "Photorefractive charge compensation at elevated temperature and application to $\mathrm{KNbO}_{3}$, J. Opt. Soc. Am. B 10, 171-185 (1993).

9. A. Yariv, S. Orlov, G. Rakuljic, and V. Leyva, "Holographic fixing, readout, and storage dynamics in photorefractive materials," Opt. Lett. 20, 1334-1336 (1995).

10. S. Orlov, D. Psaltis, and R. R. Neurgaonkar, "Dynamic electronic compensation of fixed gratings in photorefractive media," Appl. Phys. Lett. 63, 2466-2468 (1993).

11. M. Carrascosa and F. Agullo-Lopez, "Theoretical modeling of the fixing and developing of holographic gratings in $\mathrm{LiNbO}_{3}$," J. Opt. Soc. Am. B 7, 2317-2322 (1990).

12. R. Matull and R. A. Rupp, "Microphotometric investigation of fixed holograms," J. Phys. D 21, 1556-1565 (1988).

13. V. V. Kulikov and S. I. Stepanov, "Mechanisms of holographic recording and thermal fixing in photorefractive $\mathrm{LiNbO}_{3}: \mathrm{Fe}$," Sov. Phys. Solid State 21, 1849-1851 (1979).

14. P. Hertel, K. H. Ringhofer, and R. Sommerfeldt, "Theory of thermal hologram fixing and application to $\mathrm{LiNbO}_{3}: \mathrm{Cu}$," Phys. Status Solidi A 104, 855-862 (1987).

15. N. K. Kukhtarev, "Kinetics of hologram recording and erasure in electrooptic crystals," Sov. Tech. Phys. Lett. 2, 438440 (1976).

16. W. Bollmann, "Diffusion of hydrogen $\left(\mathrm{OH}^{-}\right.$ions $)$in $\mathrm{LiNbO}_{3}$ crystals," Phys. Status Solidi A 104, 643-648 (1987).

17. C. Gu, J. Hong, H. Y. Li, D. Psaltis, and P. Yeh, "Dynamics of grating formation in photovoltaic media," J. Appl. Phys. 69, 1167-1172 (1991).

18. W. Bollmann and H. J. Stöhr, "Incorporation and mobility of $\mathrm{OH}^{-}$ions in $\mathrm{LiNbO}_{3}$ crystals," Phys. Status Solidi A 39, 477-484 (1977).

19. H. Vormann, G. Weber, S. Kapphan, and M. Wöhlecke, "Hydrogen as origin of thermal fixing in $\mathrm{LiNbO}_{3}: \mathrm{Fe}$," Solid State Commun. 57, 543-545 (1981).

20. L. Arizmendi, P. D. Townsend, M. Carrascosa, J. Baquedano, and J. M. Cabrera, "Photorefractive fixing and related thermal effects in $\mathrm{LiNbO}_{3}$," J. Phys. Condens. Matter. 3, 5399-5406 (1991).

21. M. Carrascosa and L. Arizmendi, "High-temperature photorefractive effects in $\mathrm{LiNbO}_{3}: \mathrm{Fe}$," J. Appl. Phys. 73, 27092713 (1993).

22. R. Müller, L. Arizmendi, M. Carrascosa, and J. M. Cabrera, "Time evolution of grating decay during photorefractive fixing processes in $\mathrm{LiNbO}_{3}$," J. Appl. Phys. 77, 308-312 (1995).

23. O. F. Schirmer, O. Thiemann, and M. Wöhlecke, "Defects in $\mathrm{LiNbO}_{3}$-I. Experimental aspects," J. Phys. Chem. Solids 52, 185-200 (1991). 
24. T. Volk, N. Rubinina, and M. Wöhlecke, "Optical-damageresistant impurities in lithium niobate," J. Opt. Soc. Am. B 11, 1681-1687 (1994).

25. S. Klauer, M. Wöhlecke, and S. Kapphan, "Influence of $\mathrm{H}-\mathrm{D}$ isotopic substitution on the protonic conductivity of $\mathrm{LiNbO}_{3}$," Phys. Rev. B 45, 2786-2799 (1992).

26. J. R. Herrington, B. Dischler, A. Rauber, and J. Schneider, "An optical study of the stretching absorption band near 3 microns from $\mathrm{OH}^{-}$defects in $\mathrm{LiNbO}_{3}$," Solid State Commun. 12, 351-354 (1973).

27. A. Yariv, V. Leyva, and G. A. Rakuljic, "Relaxation and lifetime of 'fixed' charge holograms," in Technical Digest, 1994 IEEE Nonlinear Optics, Materials, Fundamentals, and Applications (Institute of Electrical and Electronics Engineers, New York, 1994), postdeadline paper PD6.

28. G. A. Rakuljic and A. Yariv, "Photorefractive systems and methods," U. S. patent 5,440,669 (August 8, 1995).

29. D. L. Staebler, W. J. Burke, W. Phillips, and J. J. Amodei, "Multiple storage and erasure of fixed holograms in Fedoped $\mathrm{LiNbO}_{3}$," Appl. Phys. Lett. 26, 182-184 (1975).
30. A. Mehta, E. K. Chang, and D. M. Smyth, "Ionic transport in $\mathrm{LiNbO}_{3}$," J. Mater. Res. 6, 851-854 (1991).

31. P. F. Bordui, R. G. Norwood, D. H. Jundt, and M. M. Fejer, "Preparation and characterization of off-congruent lithium niobate crystals," J. Appl. Phys. 71, 875-879 (1992).

32. D. H. Jundt, M. M. Fejer, R. G. Norwood, and P. F. Bordui, "Composition dependence of lithium diffusivity in lithium niobate at high temperature," J. Appl. Phys. 72, 3468-3473 (1992).

33. S. C. Abrahams and P. Marsh, "Defect structure dependence on composition in lithium niobate," Acta Crystallogr. Sect. B 42, 61-68 (1986).

34. U. Schlarb and K. Betzler, "Refractive indices of lithium niobate as a function of temperature, wavelength, and composition: a generalized fit," Phys. Rev. B 48, 15613-15620 (1993).

35. L. Kovàcs and K. Polgar, in Properties of Lithium Niobate, Vol. 5 of Electronic Materials Information Service Data Review Series (Institution of Electrical Engineers, London, 1989), p. 109. 